\title{
Optimising VoIP Traffic over MANET: Leveraging the Power of TORA On-Demand Routing Protocol
}

\author{
Uchenna Peter Daniel \\ Dept. Computer Science \\ Federal University Lokoja, \\ Nigeria
}

\author{
Murtala Muhammad \\ Dept. Computer Science \\ Adamawa State University, \\ Nigeria
}

\author{
Nneka C. Agbanusi \\ H. Pierson Associates Ltd, \\ Lagos -Nigeria
}

\begin{abstract}
Mobile Ad hoc Network (MANET) routing protocols studies in the last decade have focused more on proactive routing protocols with many specifics to data traffic. Only fewer works have addressed the issues of reactive protocols supporting voice application. This research work analyses the performance of VoIP application in MANET configured with Temporary Ordered Routing Algorithm (TORA) routing protocol as on-demand routing protocol. The VoIP application is evaluated using four different parameters namely; codec, MAC protocol, mobility and node density in an OPNET simulation environment. The quality of transmission was measured through different performance metrics such as jitter, Mean Opinion Score (MOS), end-to-end delay and throughput. Results show that major metrics that had high impact on the performance of the network included mobility and node density. It research proved that communication in an ad hoc network using TORA is more productive and effective with fewer nodes. It could also be said that given the utilization of TORA routing protocol in MANET, quality of voice communication is disproportionate to node density. Increase in the number of nodes greatly depreciates voice quality. In the whole, the work provides a very good analysis that could help the academia and industries in reaching decisions for a most suiting routing protocol at any given ad hoc voice communication setup.
\end{abstract}

\section{General Terms}

MANET QoS, Mobile Ad Hoc Network Routing.

\section{Keywords}

VoIP over MANET, On-demand Routing Protocol, VoIP Traffic Optimization, Mobile Ad Hoc Networks.

\section{INTRODUCTION}

The value of communication in our contemporary society cannot be over emphasized. To keep abreast with our evolving environment, an interaction via exchange of information is a reliable option. However, trends have seen rapid evolutions in modern communication systems; the wireless medium not left out. Purposefully, these recurrent changes are still bear the same singular objective of providing communication services at all times and locations, and portable computing systems have emerged as fitting solutions to this objective. Traditional approaches to network communication necessitates that a third-party infrastructure be maintained to aid efficient communication between two or more endpoints in a network. On the contrary, there are subjective circumstances that make unrealizable the traditional structure of wireless infrastructureenabled network communication. Unplanned gatherings and disaster-stricken areas are instances where communication comes critical; yet, maintaining intermediating access points would be difficult. This concern opened up a need for a system that can manage communication in an infrastructureless network environment. And as a viable answer came Mobile Ad Hoc Networks (MANETs).

Mobile Ad-hoc Network is a collection of different wireless devices connected together without the help of any network infrastructure and can be implemented within a short period of time in a small area [1]. Another definition has it as a collection of mobile nodes that can communicate with each other without the use of predefined infrastructure or centralized administration [2]. Yet another source describes it as a decentralized and autonomous network consisting of liberated mobile nodes (mobile phones, laptops, personal digital assistants (PDAs), MP3 players, and personal computers) [3]. A point common to all sources is that devices are mobile yet connected together, that is, able to maintain interaction or communication per say. Each node in the setup acts both as a host and as a router; forwarding messages to other nodes that are out of radio range [4].

Mobile Ad Hoc Network (MANET) has come-out as a product of the above necessity. It has continued to offer solution to this need. It is worthy to note that MANET just like other conventional networks, use protocols for communications. The occurrence of several types and versions of these protocols have also given rise to varying results; some efficient, others not as much. Performance has thus become a very decisive issue that requires attention. Being able to ensure efficient, uninterrupted movement of information from source to destination is germane. This can only be achieved if an apt understanding of the function features and challenges inherent in the use of the routing protocols.

The quality of service (QoS) challenge in the design of ad-hoc wireless network underwrites the development of dynamic routing protocols that efficiently find route between mobile nodes. To facilitate communication in such dynamic changing connections, varying routing protocols such as Dynamic Source Routing (DSR), Destination Sequence Distance Vector (DSDV), and Ad-hoc On-Demand Distance Vector (AODV) are introduced [1] amongst several others. These have come as adoptable solutions to the limitations and routing shortcomings in MANETs.

There are a lot of prominent identities in mobile ad-hoc network which differ from the wired network. These include dynamic topology, bandwidth constraint, variable capacity link, energy constraint operation, and limited physical security 
[5]. Different protocols have been used to test these characteristics and compare it with the routing protocols in wired connection in order to predict these variations. However, since ordinary MANET involve only data communication; the integration of voice communication will affect the routing protocols in terms of energy conservation, energy constraint, the throughput, and packet drop. In a VoIP system some sort of technique is required like voice digitalization, noise cancellation, and voice compression for the operation to be performed [6]. Given the rising swerve towards Voice-over-Internet Protocol (VoIP) with its promising paybacks, this discourse investigates the performance of voice application in a wireless mobile ad hoc network using Temporary Ordered Routing Algorithm (TORA); an on-demand routing protocol using OPNET simulator.

The light is beamed on Temporary-Ordered Routing Algorithm (TORA) to test the performance of voice communication (traffic) in Mobile Ad-hoc Network (MANET) in relations to energy conservation, efficiency, quality of service, and the packet drop. Much has been done already on the problems of duplicate address detection, merging, scalability and security breaches, even greater works have been done on general performance of routing protocols. However, not dissenting the significance of establishing communication amongst devices on ad hoc base, we submit that it is as much a concern to be able to ensure that the communication is effective. The choice of TORA is informed by its suitability for MANET protocols especially in dense population of nodes [4], its ability to maintain multiple routes from source to destination even with frequent topology changes. Its adoption of Internet MANET encapsulation protocol (IMEP) for link status and sensing neighbour connectivity, providing reliable ordered delivery of routing messages to all nodes, bandwidth efficiency and impressive adaptability to quick route repair during link failure [7]. While investigating the performances of different popular routing protocols for VoIP application in MANET scenario, TORA protocol stands out as the best candidate compared to other protocols [8].

With cognisance to the significance of time in MANET, the work is aim at suggesting a scheme implementable on TORA with the potentials for optimizing performance in the context of route request, route reply and route maintenance. In voice communication, encoding schemes have been known to determine the quality of the voice output thereof, thus, these too require consideration. As a result, various encoding schemes with speech activity factor enabled or disabled on the load, and other voice performance metrics are tested in the study. The core of our work involves an analysis that could come handy for both academia and industries (wireless equipment's manufacturing companies) when deciding protocol suitability and suiting parameter setups for any given ad hoc voice communication setup.

The performance of TORA as a hybrid and reactivedominated protocol will be tested against notable performance metrics (parameters). This is with a view to discovering the most critical metric that exerts down-beating effects on MANET with respect to voice and other heavy data. The rest of the works are outlined as follows; Chapter 2 takes-on details covering related works on MANET routing protocol classifications, routing concept with specifics to TORA, VoIP and Quality of Service of Voice packets over MANETS. Chapter 3 gives details about the system being modelled and the simulation setup (parameters and metrics). Chapter 4 presents an analysis of the simulation results while chapter 5 presents the concluding parts of the work with some recommendations and(or) future work areas.

\section{RELATED WORKS}

Mobile Ad-Hoc Network (MANET) describes a rapid deployment of communication medium between nodes in a temporary environment without third-party physical infrastructure. Information routing between nodes depend on the neighbouring nodes that are constantly in motion, maintaining a dynamic topological nature which as a result, present several functional setbacks ranging from packet loss due to transmission error, dynamic change of topology, scarce bandwidth, energy-constraint (short battery life and limited capacity), to limited physical security which serve as the natural characteristics of MANET [8]. Conventional network environment protocols (OSPF, RIP, IGRP, and EIGRP) are unable to manage such challenging scenarios hence the need for suiting alternative. To this end, several routing protocols have been proposed and analysed for MANETs. These protocols have been broadly classified into three (3); the Proactive, the Reactive and the Hybrid Routing Protocols [9].

\subsection{The Proactive Routing Protocols:}

The Proactive routing protocols keep visiting each and every node in the Network by sending Hello Messages to the nodes. The frequent change in topology would usually amount to a high cost of network maintenance. Therefore when there is a need for a route to a destination; such route information will immediately learn and adapt the changes without affecting the transmission process. The following are proactive routing protocols: Wireless Routing Protocol (WRP) [10], Destination-Sequenced Distance-Vector Routing (DSDV) [11], Optimized Link State Routing Protocol (OLSR) [8], Fisheye State Routing (FSR), Global State Routing (GSR) [12], Hierarchical State Routing (HSR) and Topology Broadcast Reverse Forwarding (TBRF) [9].

\subsection{The Reactive Routing Protocols:}

The reactive routing protocols are centred on request transmission processes also referred to as Query-reply dialog protocols. Protocol reaction emerges only when there is a request or demand by a node in the network, and it does not require any periodic transmission of topological information through the network. These protocols are sometime referred to as on-demand routing protocols [13]. The following are reactive protocols: Ad-Hoc On-Demand Distance Vector Routing (AODV) [14], Dynamic Source Routing (DSR) [15], Admission Control enabled On-demand Routing (ACOR), Associativity-Based Routing (ABR), Location-Aided Routing $(L A R)$, and Temporally-Ordered Routing Algorithm (TORA) [8].

\subsection{Hybrid routing Protocol:}

Hybrid routing protocols are protocols that are both reactive and proactive in nature. Schematic nature of protocols was aimed at increasing scalability of MANET routing protocols in order to allow neighbouring nodes of the same quality to form a backbone capable of reducing route discovery overhead [9]. Reactive protocols are more scalable than proactive protocols because of their architecture. 
Consequently, existing hybrid protocols are mostly zone based; in the sense that the network is partitioned or seen as a number of zones by each node [9].The following are examples of hybrid routing protocols: Zone Routing Protocol (ZRP)
[16], Zone-Based Hierarchical Link State (ZHLS), Scalable Location Update based Routing Protocol (SLURP) [16], Distributed Spanning Trees-based routing protocol (DST) [17] and Distributed Dynamic Routing (DDR) [18].

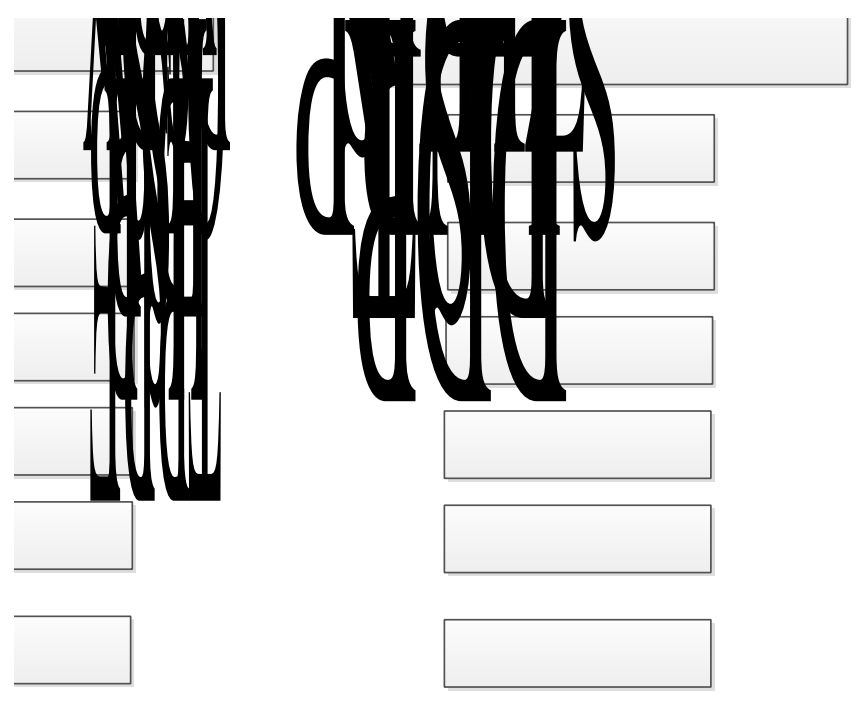

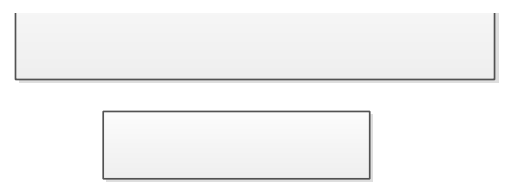
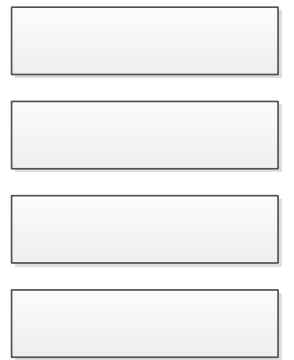

Figure 1: MANET Routing Protocols

\subsection{Routing in MANET}

One of the significant challenges in MANET is developing support for routing [19] because of the combination of different characteristics in the network such as frequent change of topology, lack of infrastructure in place to aid the communication and the movement of the nodes in the network. The biggest challenge that causes high frequent route failure in a dynamic network with rapid topological changes is mobility [20]. Any routing protocol developed to support this type of communication should be bandwidth-efficient and must produce low overhead to deliver good overall performance [19]. The routing protocols designed for wired networks are not capable to perform in MANET due to their incapacity of producing low overhead to speed up the communication which leads to different proposals for optimizing different protocol to suit MANET [21]

Routing in MANET can be classified into two different categories, which are; Unicast and Multicast routing [22]. Both embrace some form of flooding techniques to deliver a packet from source to destination. Flooding which is referred to as network-wide broadcasting [23] is a process or method of delivery data from one node to another in a communication environment. It uses MAC protocol layer as a broadcast mechanism to transfer packet from a source node to the neighbouring nodes. Broadcasting information has to be once then it will re-broadcast through another node and terminate when every node has heard the packet in order to overcome looping problem. The purpose of using flooding techniques is to eliminate the redundant broadcast and to ensure that all the nodes in the network participate actively [24].

While Unicast routing is the process of maintaining a single route in a network between all pairs of nodes regardless of whether all routes are actually used; Multicast routing referred to single source with multiple destinations to deliver a packet in a network environment [25]. Unicast routing technique is highly significant because it maintain a shortest path to destination. All proactive routing protocols are unicast routing in nature and are classified into distance vector and link state protocols. Examples of unicast routing protocols are Destination-Sequence Distance-Vector (DSDV), Wireless Routing Protocol (WRP), Optimized Link State Routing (OLSR), and Topology Broadcast based on Routing-Path Forwarding (TBRPF) [25].

\subsubsection{TORA Routing Protocol And Benefits}

Like noted in figure 1 above, TORA is a reactive driven protocol and on which our study id focused. It is clear that devices in MANET are self-configured; and do not require any administrative concern for nodes in the network to send and receive information. Conversely, communication only comes successful when every node takes-on a router responsibility on its own [26]. TORA is a reactive protocol 
which belongs to link-reversal algorithm family, which due to its scalability, efficiency and adaptability is seen adoptable in large, dense mobile networks [9]. TORA is noted to display the following capabilities; (i) it establishes routes quickly (ii) it executes in distributive mode (iii) it provides loop-free route (iv) it provides multiple routes and (v) minimized communication overhead by localizing algorithmic reactive to topological changes when possible [9].

And given the field application being considered (airport base), several environmental challenges are expected relative to changes in the network structure, communication strength (quality) and efficiency of communication (bandwidth). TORA as an on-demand protocol is not commonly used like other on-demand protocols (AODV \& DSR). TORA possesses some advantages over other reactive protocols like bandwidth efficiency, adaptability and quick route repair during link failure, and providing multiple routes to destination nodes in wireless networks [27]. TORA limits control message propagation in highly dynamic mobile environment which is the main objective behind the establishment of the routing protocol, and it is a destination oriented protocol that supports both reactive and proactive routing [13]. It is designed to work towards high reactivity rather than routing optimality only in a high dynamic mobile environment because of its flexibility and that is its primary and secondary mechanism that supersedes other on-demand protocols [27].

\subsection{VoIP and Its Characteristics in MANET}

The development of Voice over Internet Protocol (VoIP) led to low cost telephony services. However, in a Mobile Ad-hoc Network, it is one of the heavy applications that have led to some challenges in the performance of the network, precisely because of the mobility of the nodes [28]. VoIP implementation on MANET platform is easy [29] and the challenges can be trounced with the used of strong routing protocols. Efforts however have been made to improve the performance of MANET before they can be used for VoIP purpose. VoIP is a practical scenario that can be studied to validate the performance of MANET using different routing protocols in a simulation environment, because it provides different support for simulating Constant Bit Rate (CBR), Transmission Control Protocol (TCP), ad-hoc routing, and multicast protocols over wired and wireless networks. Performance evaluation of any MANET routing protocol that carries voice traffic depends on the type of coders used in the communication setup, and the factors to be considered are complexity, algorithm delay, and bit rate quality [30]. Also, the impact of any matrix on voice communication quality depends on the codec used [31].

\subsubsection{Quality of Service of VoIP in Mobile Ad-hoc Network (MANET)}

Mobility is the basic challenge of MANET in VoIP context [29]; this affects the structure of the network and throughput of the transmission. And though there is no specific routing protocol that is best for heavy application, packet loss ratio in VoIP context is less compared to other applications because VoIP is tolerant for small packet losses [32]. The quality requirements of porting telephone conversation over a MANET are vulnerable to variations in some network parameters; end-to-end delay and packet delivery ratio due to the mobility of the nodes. Simulation result shows point-topoint voice transmission between two (2) nodes to also be factors without considering high density of the network [33].

The Real-time Services over MANET is one of the significant factors to be considered too, especially the voice communication in the network [34]. Quality of Service (QoS) also depends on various parameters, which include node speed, number of connections and area covered by the network. The implementation of a VoIP test-bed on a multihop IEEE 802.11b wireless ad-hoc network depends on the nature of the nodes in the network; could be either stationary or dynamic. However, VoIP transmission using AODV \& OLSR routing protocols performs better when the nodes are in stationary mode [35]. But when the nodes are in dynamic mode, there is rapid fluctuation of signal (jitter) in the transmission a resolution of which involves the utilization of a hybrid routing protocol, since it is capable of boosting the performance of the network in terms of QoS.

Research has shown that greater success is predicted in Mobile Ad-hoc Network which may offer a good platform for the fast deployment of VoIP mobile [36]. One limitation observed was related to the high variability of the network topology and channel behaviour which heavily influenced the service quality due to route losses and the significant delay variations. To suggest a scheme for fixing such impairments, the source is responsible for selecting the transmission path and adjusting the play-out delay with adaptive inter-talks part approach [36]. OLSR was used in collecting the statistics of the network stations which relies on the quality of the routing algorithm, and voice is measured by the means of the ITV-TE model while the nodes are in a stationary mode. Considering AODV as reactive routing protocol and OLSR as proactive routing protocol still in terms quality of service, it was discovered that AODV performs better in a very hostile medium while OLSR present better results when more mediums take place [28].

Researchers investigated the issues to be considered when implementing VoIP in terms of the size and management. The management issue derived included; performance, reliability, availability, scalability, obsolescence \& service life, regulatory issues, electricity consumption of VoIP network equipment, backup equipment, backup power, backup phones, and Bandwidth. The conclusion was that VoIP requires much more electricity to operate which can affect the performance of the network [37]. An adaptive control scheme was used to improve the quality of voice transport over wireless ad-hoc network which also has to do with the voice coding bit rate to increase the bandwidth so as to maximize the voice quality [38].

MANET topology is dynamic in nature which also affects the energy of the nodes in the network (energy conservation and constraints). Nonetheless, to control these topological issues, various techniques have been proposed by researchers. These techniques include Cone-Based Distributed Topology Control (CBTC), Ant-Based distributed, Local Information No Topology (LINT), Local Information Link Topology (LILT), Mobile-Grid topology, flat-grid topology, and Point-to-point (P2P) network topology. To control the topology of the network the size of the network also matters, because if the network is of high density therefore controlling the topology will be difficult. Researchers concentrated on energy efficient topology control, paying more attention on construction and maintenance of a network topology with good connectivity 
and minimum power consumption. The topology of an ad hoc network can be controlled by transmitting power and antenna directions [39], [40] and [41].

The strength of the transmission (capacity) depends on the channel medium which determines the throughput of the transmission. Throughput defines the total number of bits that are sent through the channel per second [42]. The throughput depends on the bandwidth used in sending the information which is the maximum number of bits that can be sent per second through the ad-hoc network. The audio codec affects the size of storage in nodes and also is heavy in terms of transmission unlike other applications, which as well affects the routing protocol in MANET. As deduced, MANET challenges comparative to Quality of service, Topology and the Capacity of the network show the high effect of MAC layer queuing on the routing protocols as well as on mobility model deployed. Different routing protocols are used with varied network topologies to access the overall effects of routing on load. Observations are that different protocols performed differently at high mobility while some obtained highest throughput when in static mode. The over-head also increase considerably for proactive routing protocols than for the reactive proactive [43].

\subsubsection{VoIP Applications in MANET}

By way of solution, It has been suggested that topological control that will minimize the challenges with different techniques. A robust and secure framework for voice transmission over multipath MANET has been proposed and the framework is not only on dynamically changing of the topology but also works against diverse environment [44]. Another management framework for MANET routing protocol OLSRV2 and constituent protocol NHDP is based on the Simple Network Management Protocol (SNMP) [45]. Thus, the paper details the various Management Information Bases (MIBs) for router status monitoring and control. Therefore, monitoring the performance and setting configuration parameters is important in order to ensure proper turning and maintenance of MANET [45]. Through experimental tests [46], researchers has also sought to discover the impact of encryption mechanisms on voice speech quality in widely deployed wireless technology 802.11 and Bluetooth. Moreover, he also evaluates the upper bound on number of simultaneous VoIP calls which can be placed and user's computational model E-model to assess the quality of service [46].

\section{SYSTEM MODELLING}

The simulation system consists of five (5) components integrated together to form a working system. These include; Ad hoc device/nodes, Ad hoc Network, a VoIP server, a VoIP Application/Profile and the VoIP Mobility model. The simulation system is structured to replicate the form of a real life MANET environment. Adopted nodes include handheld mobile phones, vehicle-attached phones and laptops, assuming an airport conversational environment. The choice of devices is informed by the key requirement that nodes within this network be in continual motion (mobility).

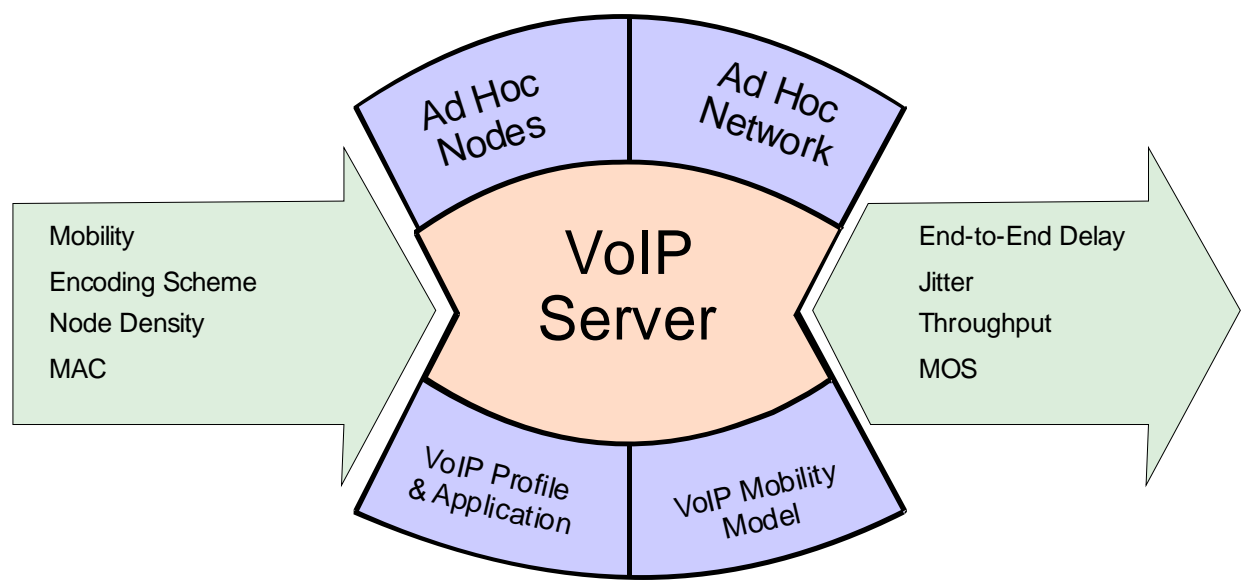

Figure 2: System design diagram

As seen in figure 2, the Ad hoc nodes (Mobile phone or laptop) in this network functions as clients that bear the task of sending requests to the network and receiving same when necessary. The Ad Hoc Network is evaluated by monitoring the VoIP traffics that transverse through the channel for the exchange of traffic data between ad hoc nodes. The TORA routing protocol determines the mode in which the exchange is carried out. A VoIP server is an independent node that aids the communication between other mobile nodes and the application configuration node. However, this is optional. The VoIP application and profile maintains information about the application being tested, and its attributes. The application configuration node maintains application layer related parameters that can be used by all nodes in the network. A mobility model configuration is introduced to help define the speed, pause time, start time and stop time of all the nodes in the network. 


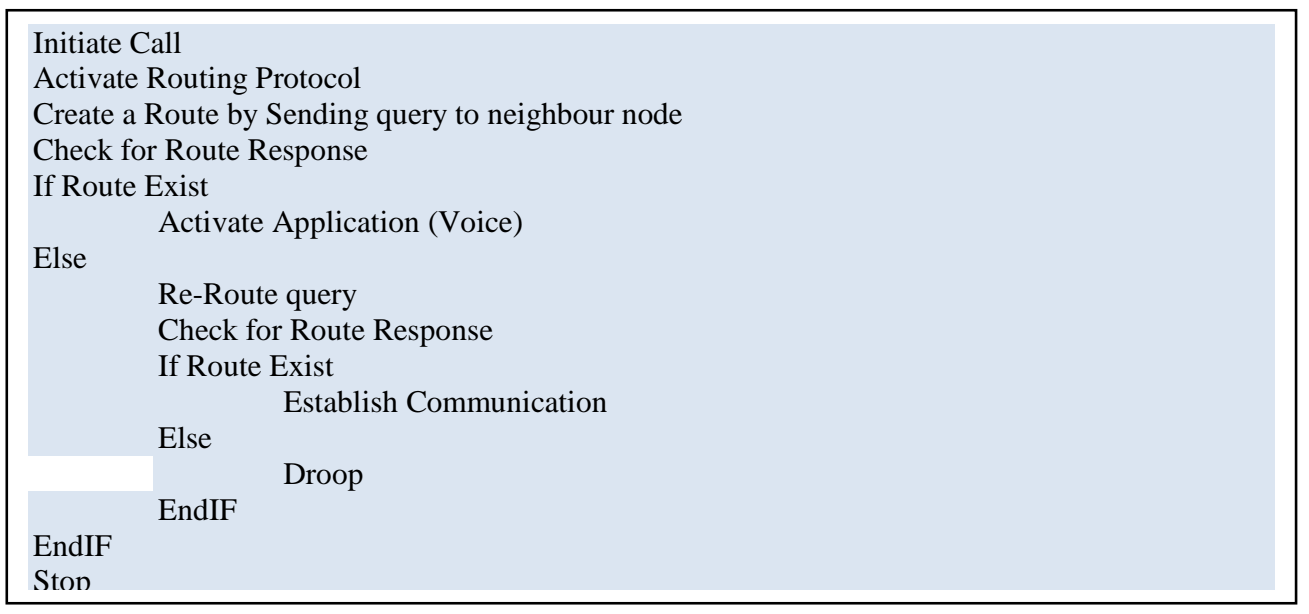

Figure 3: Pseudocode showing the process flow

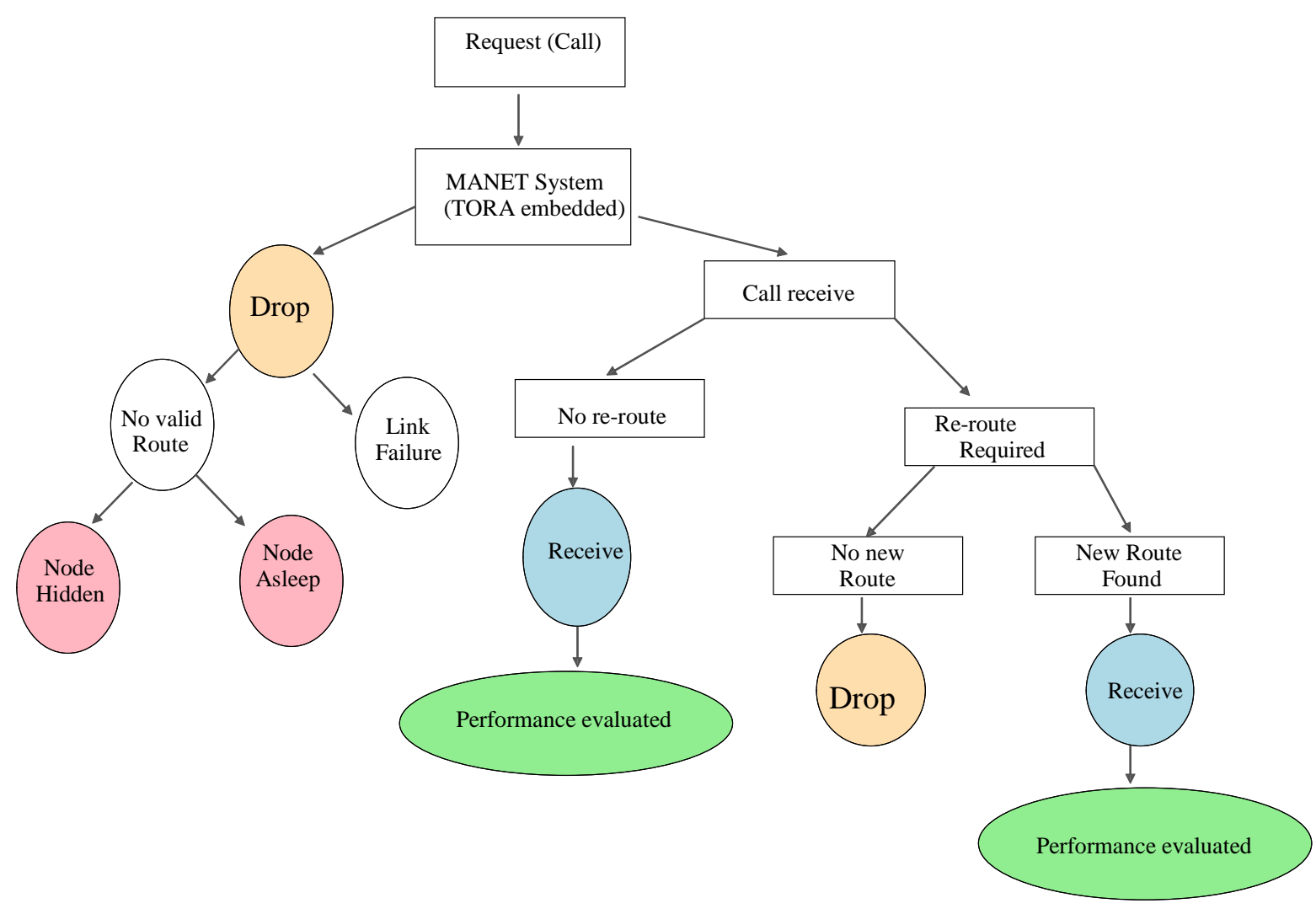

Figure 4: Process flow diagram

The concept as presented above maintains that a minimum of two route initiations are carried out before any termination. This is as opposed to the traditional routing protocols that terminate route after initial route failure. The conceptual model finds basis on the verity that on-demand routing protocols establish paths only when necessary. A route establishment operation invokes a route-determination procedure. Such procedures only terminate when either a route has been detected or no route is available after examination of all potential route paths.
The process flow for TORA routing protocol is in three stages. These include; route creation, route maintenance and route erasure. When call is initiated, it will be dropped, received or re-routed. It would be dropped based on two conditions; either no valid route (if the nodes are in sleep mode or hidden due to less energy to participate) or when there is a link failure in communication. However, if a call is received, then performance can be evaluated or there would be a tendency of re-routing. 
Table 1: Simulation Setup

\begin{tabular}{|c|c|}
\hline Statistics & Value \\
\hline Simulator & OPNET 14.5 \\
\hline Routing Protocol & TORA \\
\hline Application Traffic & VoIP \\
\hline Network Area & 500 x 500 meters ( 100 nodes) \\
\hline Simulation Time & 12 hrs. (43200 secs) \\
\hline Test Parameters & $\begin{array}{ll}\text { - } & \text { Mobility } \\
\text { - } & \text { Node Density } \\
\text { - } & \text { Encoding Scheme (Codec) } \\
\text { - } & \text { MAC Protocol } \\
\end{array}$ \\
\hline Test Metrics & $\begin{array}{ll}- & \text { End-to-End Delay } \\
\text { - } & \text { Jitter } \\
\text { - } & \text { Throughput } \\
\text { - } & \text { Mean Opinion Score (MOS) }\end{array}$ \\
\hline
\end{tabular}

Table 2: Simulation Scenarios

\begin{tabular}{l|l|rl}
\hline \hline Scenarios & $\begin{array}{l}\text { Number of } \\
\text { Scenarios }\end{array}$ & \multicolumn{2}{l}{ Description } \\
\hline Default & 1 & Is a default (starting) scenario \\
\hline Encoding scheme & 4 & - & G.711 with speech activity enable \\
& & - & G.711 with speech activity disable \\
& & - & G.729 with speech activity enable \\
& 3 & - & G.729 with speech activity disable \\
\hline Mobility & 3 & - & $20 \mathrm{~m} / \mathrm{s}$ \\
& & - & $30 \mathrm{~m} / \mathrm{s}$ \\
\hline MAC Protocol & 2 & - & IEEE $802.11 \mathrm{~b}$ \\
& & - & IEEE $802.11 \mathrm{~g}$ \\
\hline Node Density & 4 & - & $100 \mathrm{nodes}$ \\
& & - & 50nodes \\
& & - & $25 n$ ndes \\
\hline \hline
\end{tabular}

\subsection{System Setup}

The performance evaluation of MANET routing protocol with VoIP traffic running depends vastly on the workload defined in the simulation environment. As shown in table 1 (System model), the default setup consist of a network area of $500 \times 500$ meters with one hundred (100) nodes placed at random and in random motion. The default encoding scheme for the simulation is G.711 codec with a packet size of 200bytes placed on each node in the network for transmission randomly. The starting time of the transmission is set $0.0 \mathrm{sec}$ with constant mobility of $10 \mathrm{~m} / \mathrm{s}$. The length of the simulation was set to be $12 \mathrm{hrs}$ after setting the remaining scenarios. The starting $12 \mathrm{hrs}$ of the simulation will allow the system to attain a certain level and sustain a steady state. This is in line with requirements for every simulation environment, that a model requires approximately 20secs as warm-up period [47].

This study takes-on a limited test parameters; mobility, codec type, node density and MAC protocol. This limited choice is influenced by time constraints against the elongated time that would be required in performing an exhaustive performance on all parameters. The metrics for test are end-to-end delay, jitter, throughput and Mean Opinion Score (MOS). All of these features are necessary to determine the performance of TORA-routing protocol in a mobile Ad Hoc voice communication network. 


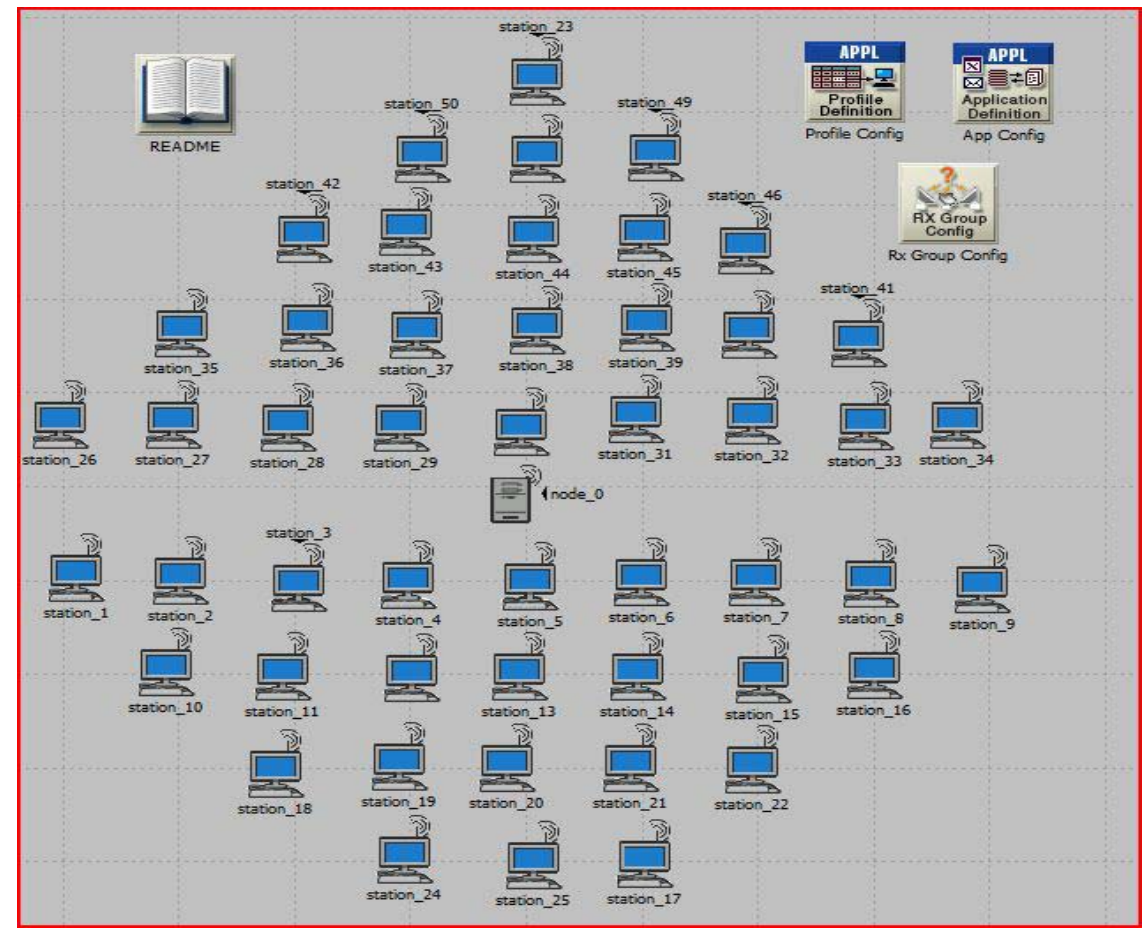

Figure 5: Network Topology

\section{ANALYSIS OF RESULTS}

Given the OPNET setup described earlier, and having conducted the simulation therein, factors looked into during the test included; mobility, node density, encoding schemes and MAC protocol for which test metrics of end-to-end delay, jitter, throughput and Mean Opinion Score (MOS) were carried out. The aim remained the test for the performance behaviour of TORA-enabled mobile ad hoc network transmitting voice traffic. Below are the results obtained and their analysis.

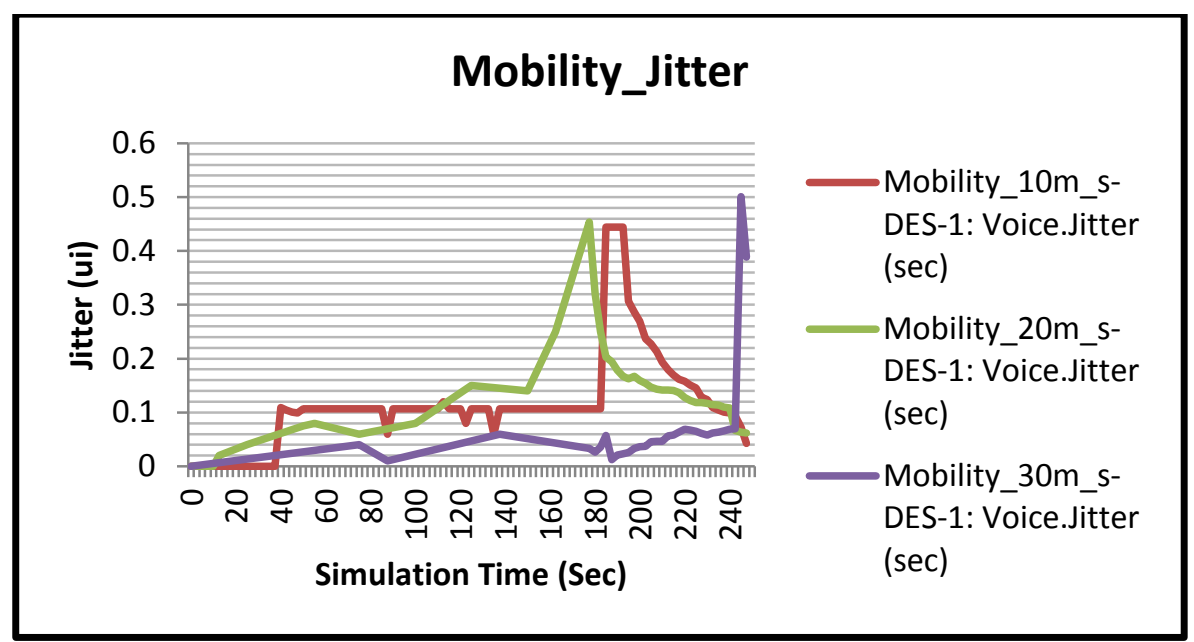

Figure 6: Mobility Jitter 


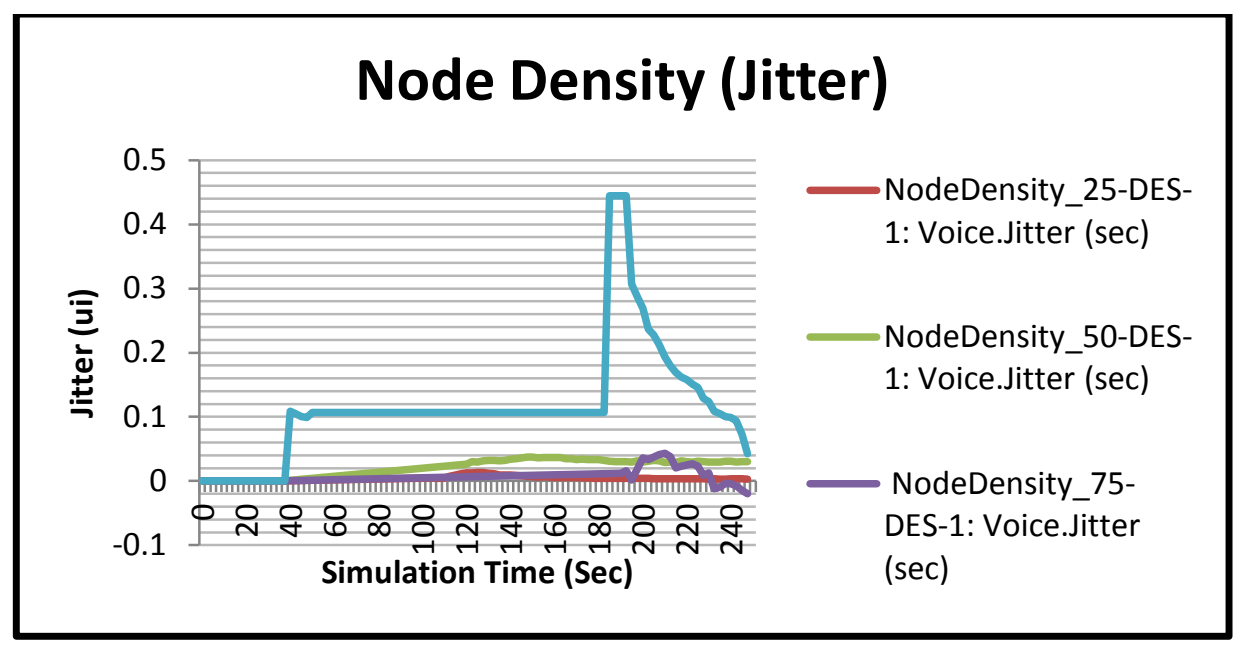

Figure 7: Node Density Jitter

\subsection{Jitter Analysis}

It is noted in the default scenario that, the jitter of voice communication in the MANET using TORA routing protocol went less than 0.1 sec as communication time increased and it reached its peak level between 75 to 85 seconds. This implies continuous communication. MAC layer protocol also has no impact on voice jitter. Both MAC layer protocol 802.11b with encryption and $802.11 \mathrm{~g}$ with encryption did not affect the network. Thus, the encoding scheme did not alter the behaviour of the ad hoc network. G.711 and G.729 encoding schemes all maintained similar output with the default scenario which implied that jitter in the network tended to its minimum regardless of the codec deployed; hence voice quality was not tampered with.

However, mobility is seen to have significant impact on the behaviour of the ad hoc network, especially as the nodes move faster. Given that the nodes were randomly placed, a mobility speed of $20 \mathrm{~m} / \mathrm{s}$ increased the jitter from initial 0.1 to 0.45 . It means that increase in mobility speed also increases the jitter as seen in figure 6. High jitter influence due to increased mobility amounts to an increase in the Bit Rate Error (BRE) in the network. Usually, the movement of nodes causes a reduction in message periodically, most glaringly; nodes receive a great deal of routing traffic above normal at mobility, thus reduction in periodicity will exert overhead in the ad hoc network. Therefore, jitter is directly proportional to mobility speed in a MANET, because as speed increases the jitter also increases.

In addition, Node density is another parameter that does affect ad hoc network performance as shown in figure 10. It is clearly shown on figure 7 that Node density is directly proportional to Jitter. Mathematically represented as;

$$
\mathrm{D} \alpha \mathrm{J} \text {, Therefore, } \mathrm{D}=K J
$$

Where $\mathrm{D}$ is the Node density, $\mathrm{J}$ is the Jitter and $\mathrm{K}$ is the constant of proportionality. Thus, the lower the density, the lower the jitter and hence the higher the quality of the communication and Vice-versa. Therefore, reduction in the node density impacts the network by causing a reduction in jitter.

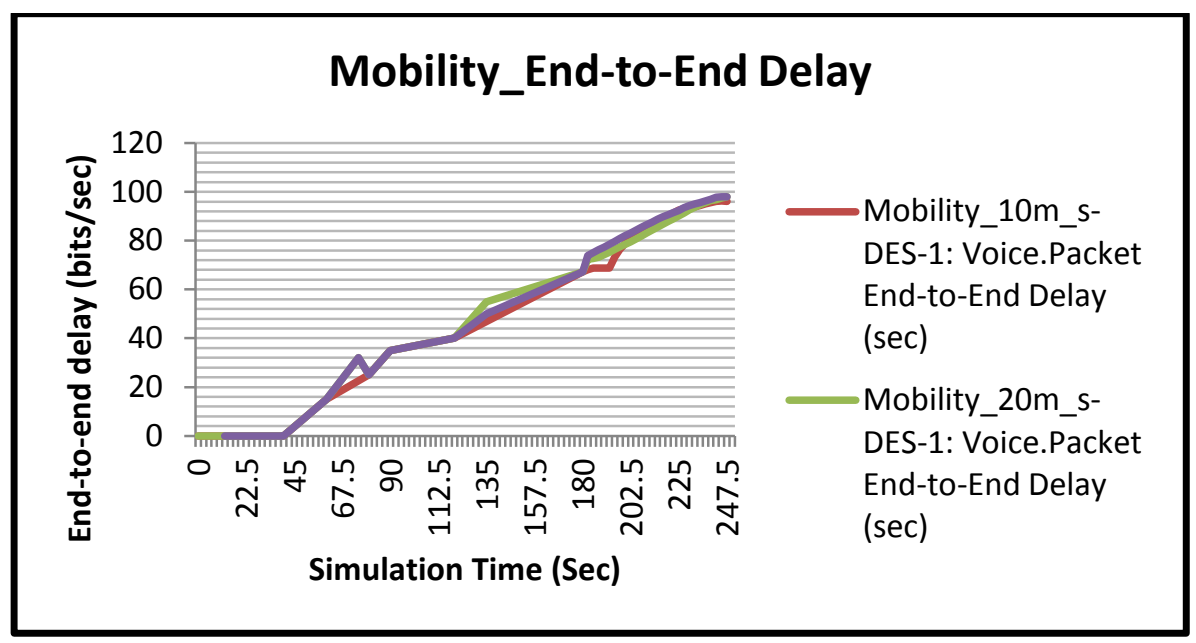

Figure 8: Mobility End-to-End Delay 


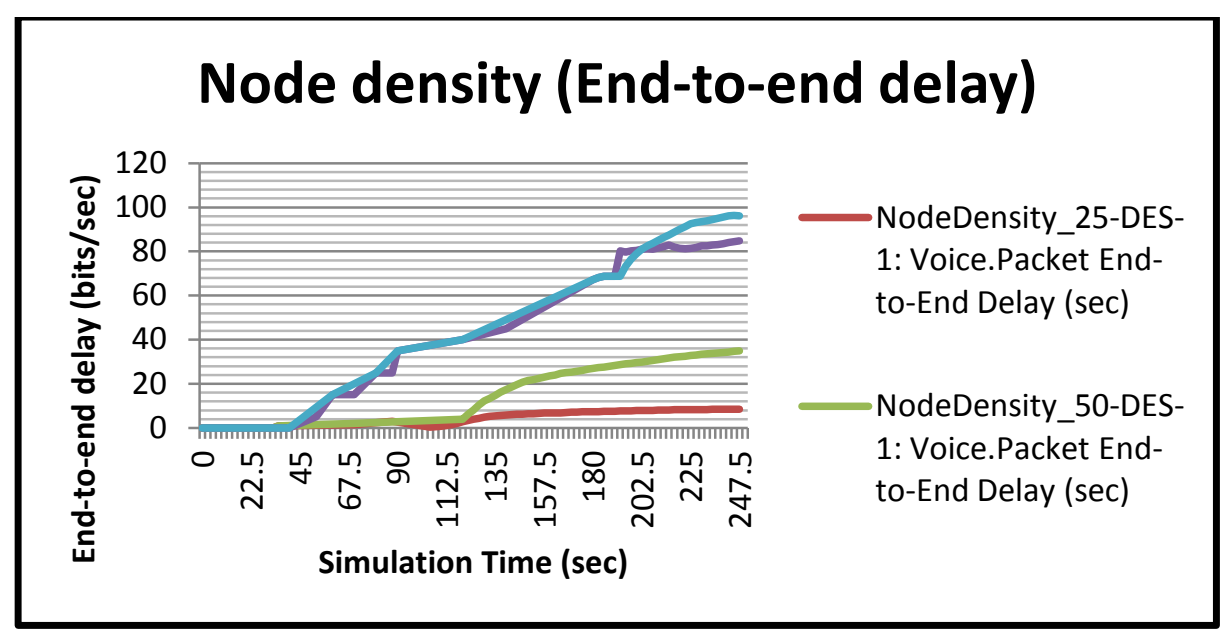

Figure 9: Node Density End-to-End Delay

\subsection{End-to-end Delay Analysis}

It is observed that end-to-end delay of a network with node density parameters starts at about 40 seconds and rises to about 96 seconds with a short time of communication. This remained unchanged even with the change in MAC layer protocols and (or) variations in encoding scheme. All these parameters maintain an average end-to-end delay that is consistent with standard recommendations of an average of 150ms for an unnoticed end-to-end delay.

While significant impact is noticed in the mobility (figure 8) and Node density (figure 9) scenarios. It is noticed that endto-end delay status is maintained for mobility value at $10 \mathrm{~m} / \mathrm{s}$ and less. However, the delay is seen to begin to drop slightly as the mobility increases to $20 \mathrm{~m} / \mathrm{s}$ and $30 \mathrm{~m} / \mathrm{s}$. Although packets reach delay of above $60 \mathrm{~m} / \mathrm{s}$, it is considered that transmission of data and traffic area beat faster than in the default.

Node density also exerts severe impacts to network end-to-end delay as noted earlier. A density of 100 nodes yields a delay near to the initial scenario with no impact; however, as the nodes number reduces the delay also tends to reduce. It implies that spaces allow for reduced collision of nodes, allowing for route establishments and (or) re-establishment of route and acknowledgement.

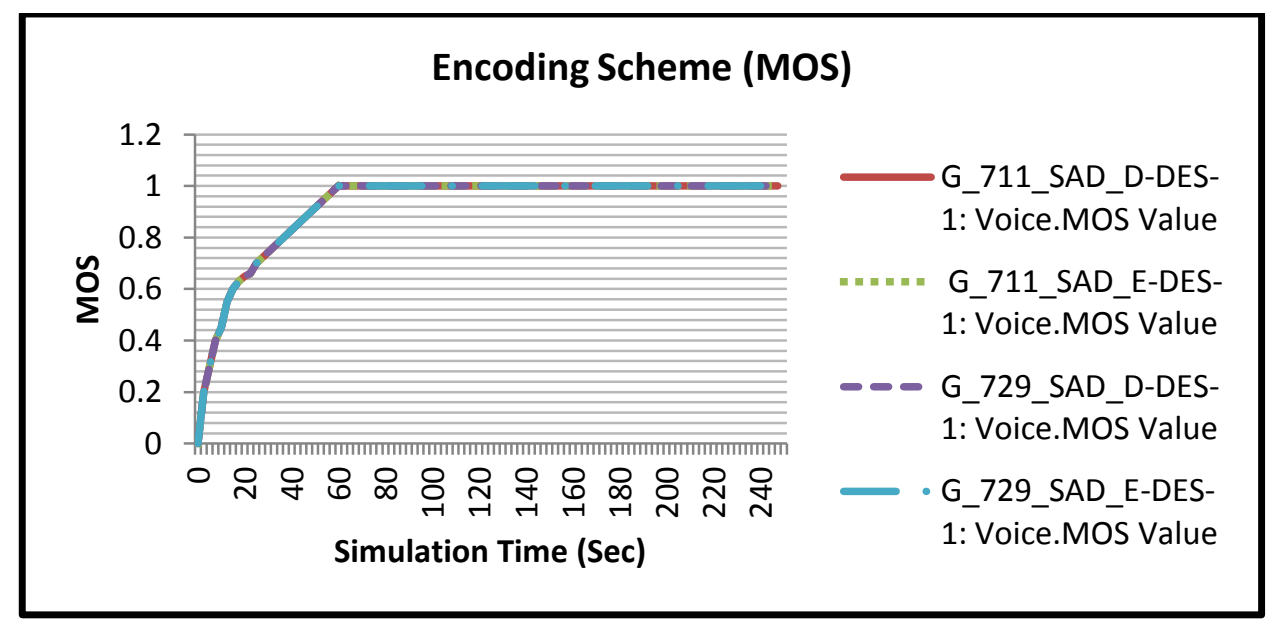

Figure 40: Encoding Scheme MOS 


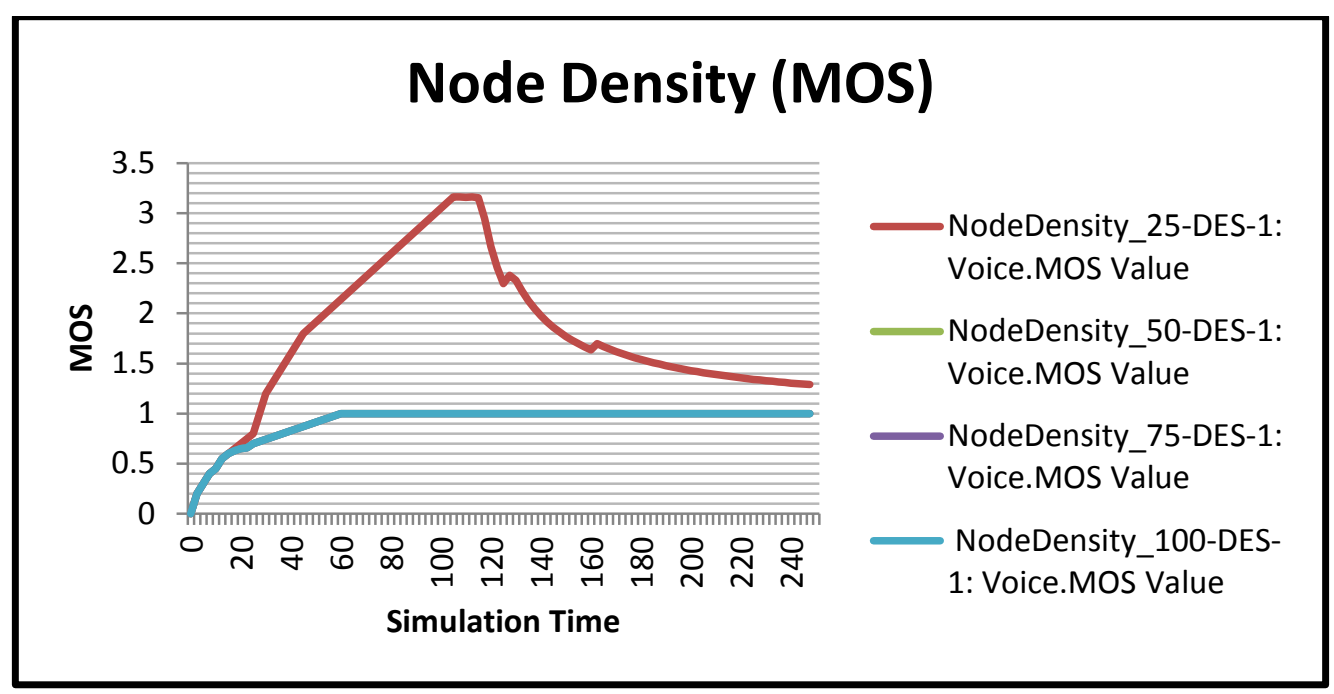

Figure 51: Node Density MOS

\subsection{Mean Opinion Score (MOS) Analysis}

Mean Opinion Score (MOS) factor maintains a stable value of 1 from 70 seconds. This value is replicated in the MOS values obtained while evaluating the performance of the network with ranging encoding schemes, as shown in figure 10. G.711 and G.729 with and without Speech Activity Detection (SAD) all have stable MOS value of 1 . Mobility scenarios also yielded the same value irrespective of node speed. A change in MAC protocol layer also does not significantly alter the voice quality. A voice quality (MOS) of 1 indicates that the nodes are not able to establish and maintain communication (nearly zero traffic), implying 'impossible or poor communication' as defined by ITU-TP 800 scale.

Slight changes are noticed in node density scenario shown in figure 11. While node densities of 50, 75 and 100 still maintained poor communication quality with a MOS value of 1. A node density of 25 yields a MOS of about 3.2; implying a fairly annoying quality, but better than when it is impossible.

In this case the node density is inversely proportional to the MOS unlike in the Jitter. The higher the node density the lower the MOS and the other way round. The voice quality rating here is classified as low. This means that communication in an ad hoc network using TORA is more productive and effective with fewer nodes and given wider space range between nodes. Increase in the number of nodes greatly deteriorates voice quality. As the environment or system gets dense with nodes, the quality of voice communication degrades. However, there is a point where communication is hardly to take place because of small value of MOS in the network especially in scenarios that have high dense nodes (50 to 100 nodes).

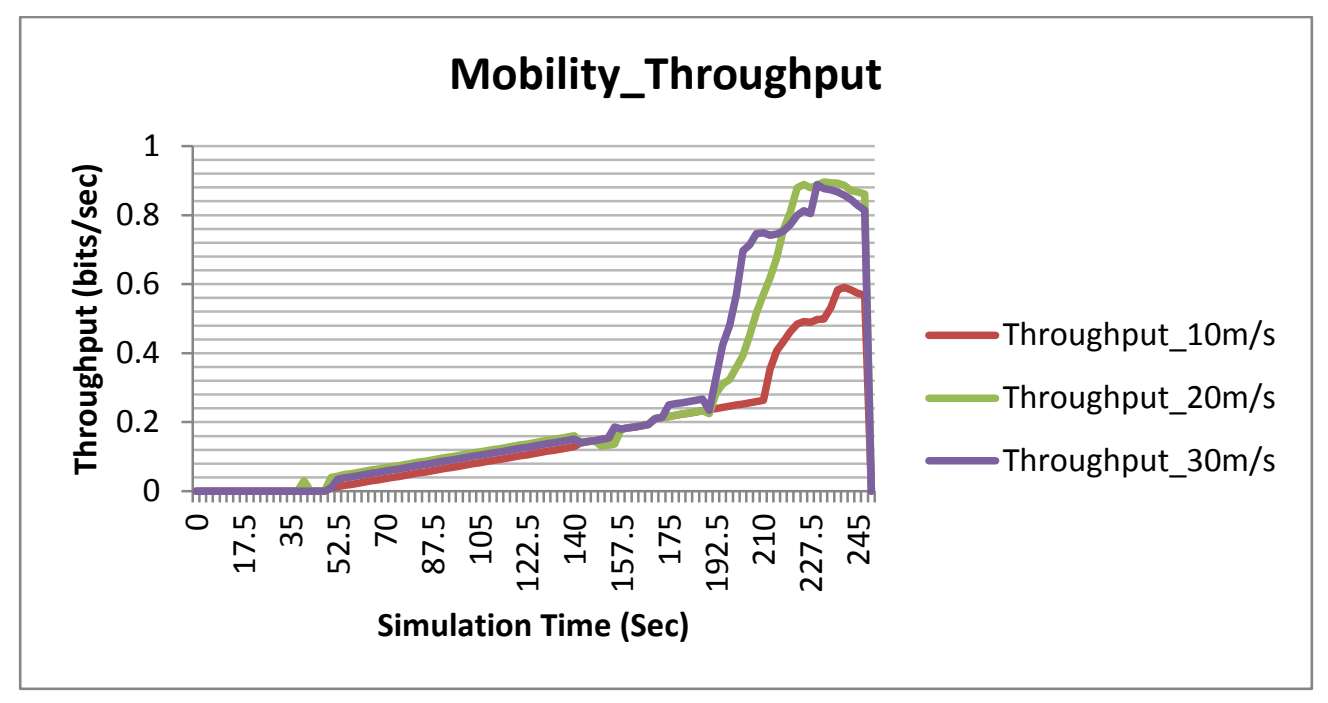

Figure 62: Mobility Throughput 


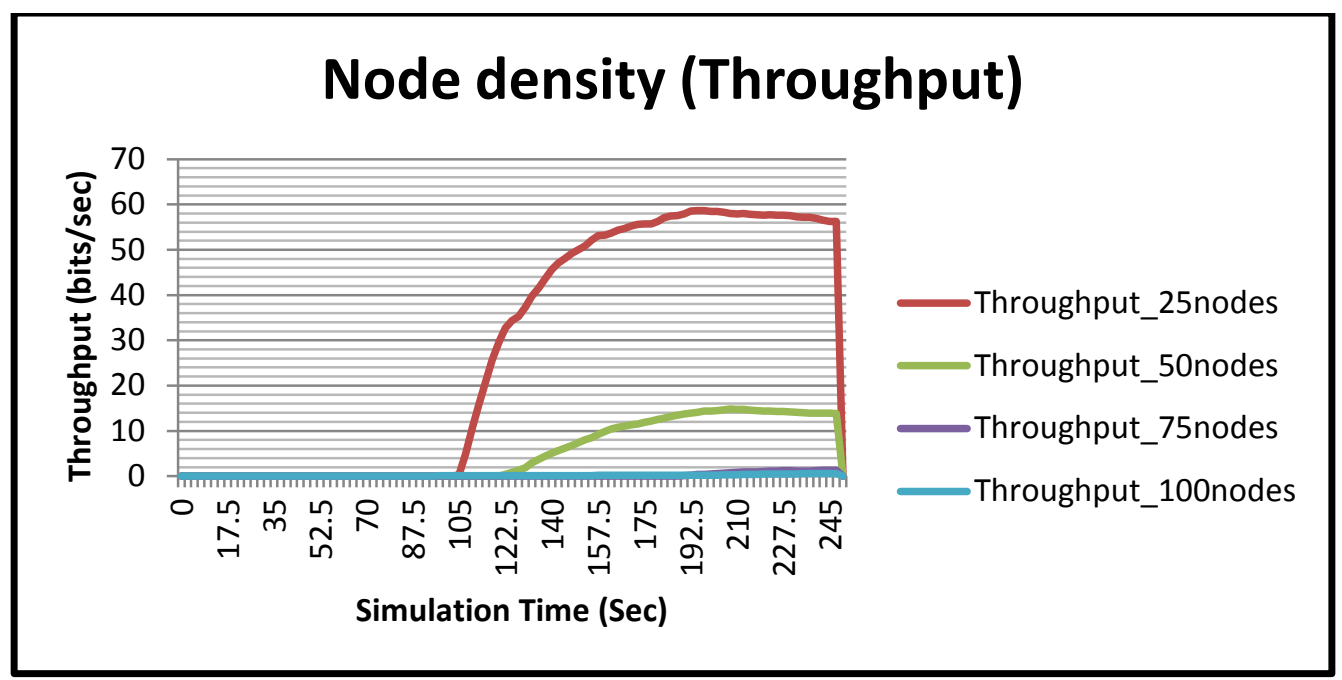

Figure 13: Node Density Throughput

\subsection{Throughput Analysis}

From the default setup, network throughput rises to a maximum of $0.6 \mathrm{bit} / \mathrm{sec}$ more than halfway the transmission period. However, a drastic drop is also noticed as the time of transmission increases. Change in encoding schemes does not affect this behaviour in any way, and the encryption features do not make the situation any better. Changes in the MAC layer protocols also do not alter the behaviour. A maximum throughput of $0.6 \mathrm{bits} / \mathrm{sec}$ is still maintained all the way.

Unlike MAC layer protocol and encoding scheme, mobility status is seen to affect the behaviour of TORA in the ad hoc network. An increase in mobility triggers early throughput emergence and increases the overall throughput results. A nearly $50 \%$ increase is experienced as the mobility doubles. Figure 12 shows that a $20 \mathrm{~m} / \mathrm{s}$ mobility rates yield a throughput of about $0.9 \mathrm{bits} / \mathrm{sec}$ as well as the $30 \mathrm{~m} / \mathrm{s}$ mobility rate. With such mobility results, node density is also seen to affect the behaviour of TORA routing protocol. However, the Node density relationship takes-on a reverse proportionality. Figure 13 shows that the higher the node density, the lower the throughput and vice-versa. We thus, model the behaviour as follows;

If $\mathrm{N}$ represent the Node density and $\mathrm{T}$ the throughput, then the relationship will be denoted mathematically as follows;

$$
\mathrm{N} \alpha \frac{1}{\mathrm{~T}} \quad ; \quad \text { Then } \mathrm{N}=\frac{K}{T}
$$

Increase in node density diminishes the throughput, and at a point no traffics or packets are successfully delivered. Even though there are signs of potential drop in throughput with time, the fewer the nodes the easier and better communication will be achieved.

\section{CONCLUSION AND FUTURE WORK.}

Mobility and the Node Density are key factors that upset the behaviour of TORA-enabled Mobile Ad hoc Network. TORA has three stages of operations (route creation, route maintenance and deletion); the time taken for route reply is not specified in the route creation stage. The conceptual model presented finds basis on the fact that on-demand routing protocols establish paths only when necessary. The route establishment operation invokes a route-determinations procedure. However, such procedures only terminate when a route is been detected or no route is available. Therefore, specifying the time taken for the reply to occur might optimize the performance of TORA with respect to mobility and node density. The time it takes to establish communications is as quite important, and the suggested model adapts a four (4) seconds benchmark. Standards emphasize on seven (7) seconds limits, which could be measured. However, most ad hoc routing protocols have been noted to have matched this requirement.

Jitter is seen to be severely impacted by the mobility of the nodes. Moreover, the results also indicate that an increase in speed of the nodes on the network influences an attendant increase in bit rate error. Node density also causes strict impact on network end-to-end delay. A higher number of nodes are seen to aggravate the delay such that packets cannot be transported effectively. However, as the nodes number reduces, the delay tends toward reduction, allowing for efficient communication among nodes. Although general voice quality is not very encouraging, fewer number of the nodes within the network make better results an average of 3.2 MOS value is obtained which is considered fair. This means that communication in an ad hoc network using TORA is more productive and effective with fewer nodes. It could also be said that given the utilization of TORA routing protocol in MANET, quality of voice communication is disproportionate to node density. Increase in the number of nodes greatly depreciates voice quality. Overall throughput is also influenced by the mobility and density of nodes.

Given the airport conversational environment assumed, future work could analysis of other on-demand routing protocols with VoIP application and with different techniques. This study focuses on only VoIP application with speech activity detection enabled/disabled, which also it reveals more systematic general understanding of how VoIP operate in MANET. The work comes useful to the academia and industries, as it would help the decisions for a most suitable routing protocol at any given ad hoc voice communication setup. Future works could consider the behaviours of other heavy multimedia applications over MANET. 


\section{REFERENCES}

[1] J Zhang, J Zou, and Q Zhao, "MANET Routing Protocol for improving Routing discovery based on AODV," in International Conference on Network Security, Wireless Communication and Trusted Computing, 2009, pp. 197-200.

[2] Y Kim, I L Moon, and S Cho, "A Comparison of Improved AODV Routing Protocol Based IEEE802.11 and IEEE802.15.4," Journal of Engineering Science and Technology, vol. 4, no. 2, pp. 132 - 141, 2009.

[3] T Camp, J Boleng, and V Davies, "A survey of mobility models for ad hoc network research," Wireless Communications and Mobile Computing (WCMC): Special issue on Mobile Ad Hoc Networking Research, Trends and Applications, vol. 2, no. 5, pp. 483-502, 2002.

[4] Sumit Mahajan and Vinay Chopra, "Performance Evaluation of MANET Routing Protocols with Scalability using QoS Metrics of VOIP Applications," International Journal of Advanced Research in Computer Science and Software Engineering, vol. 3, no. 2, pp. 150-156, 2013.

[5] Piyusha Desai and Chirag Pandya, "Secure Routing in Mobile Ad Hoc Networks: A Survey," International Journal of Engineering and Innovative Technology (IJEIT), vol. II, no. 10, pp. 145-148, 2013.

[6] S Armania, L Gallucci, A Leonardi, and S Palazzo, "Transmission of VoIP Traffic in multihop Ad hoc IEEE 802.11n Networks," in Firs International Conference on Wireless Internet, 2005, pp. 148-155.

[7] Nadia Qasim, Fatin Said, and Hamid Aghvami, "Mobile Ad Hoc Networking Protocols' Evaluation through Simulation for Quality of Service," IAENG International Journal of Computer Science, vol. 36, no. 1, pp. 1-9, Fenruary 2009.

[8] Mohammed Shaffatul Islam, Adnan Riaz, and Mohammed Tarique, "Performance analysis of routing protocols of mobile ad hoc networks for VoIP applications," Cyber Journals: Multidisciplinary Journals in Science and Technology, Journal of Selected Areas in Telecommunications (JSAT), pp. 2633, 2012.

[9] Kumar Sarkar Subir, G Basavaraju T, and Puttamadappa C, Ad Hoc Mobile Wireless Networks: Principles, Protocols and Applications. Boca Raton, USA: Auerbach Publicatios, Taylor \& Francis Group, 2008.

[10] S Murthy and J J Garcia-Luna-Aceves, "A routing protocol for packet radio networks.," in The First Annual ACM International Conference on Mobile Computing and Networking, Berkeley, CA., 1995.
[11] C E Perkins and T J Watson, "Highly dynamic destination sequenced distance vector routing (DSDV) for mobile computer," in ACM SIGCOMM' 94, London, 1994.

[12] T W Chen and M Geria, "Global State routing: A new routing scheme for adhoc wireless networks," IEEE ICC, 1998.

[13] Qasim Nadia, Said Fatin, and Aghvami Hamid, "Mobile Ad Hoc Networking Protocols' Evaluation through Simulation for Quality of Service ," IAENG International Journal of Computer Science , vol. 36:1, pp. 1-9, 2009.

[14] E M Belding-Royer and C E Perkins, "Evolution and future directions of the ad hoc on-demand distancevector routing protocol," Ad Hoc Networks, vol. 1, pp. 125-150, 2003.

[15] D Johnson, D Maltz, and J Jetcheva, "The dynamic source routing protocol for mobile adhoc networks," Internet Draft draft-manet-dsr-07, 2002.

[16] Wang Yu and Wu Jie, "Label Routing Protocol: A New Cross-layer Protocol for Multi-hop Ad hoc Wireless Networks," Journal of Pervasive Computing and Communication, vol. 1, no. 1, pp. 1-8, 2006.

[17] S Radhakrishnan, N S.V Rao, G Racherla, and C N Sekharan, "A routing protocol for ad hoc networks using distributed spanning trees.," in IEEE Wireless Communications and Networking Conference, New Orleans. USA., 1999.

[18] N Nikaein, H Laboid, and C Bonnet, "Distributed dynamic routing algorithm (DDR) for mobile ad hoc networks. MobileHOC 2000," in First Annual Workshop on Mobile Ad Hoc Networking and Computing, 2000.

[19] Pravin Ghosekar, Girish Katkar, and Pradip Ghorpade, "Mobile Ad Hoc Networking: Imperatives and Challenges," International Journal of Computer Application, no. Special Issue, 2010.

[20] S Basagni, I Marsic, V R Syrotiuk, and B A Woodward, "A Distance Routing Algorithm for Mobility (DREAM).," In Proceeding of IEEE/ACM MobiCom , pp. 76 - 84, 1998.

[21] J Sucec and I Marsic, "An Application of Parameter Estimation to Route Discovery By On-Demand Routing Protocols.," In Proceedings of IEEE ICDCS, pp. 207 216., 2001.

[22] S R Das, R Castaneda, and J Yan, "Simulation-based Performance Evaluation of Routing Protocols for Mobile Ad hoc Networks.," ACM/Baltzer Mobile Networks and Applications (MONET), vol. 5(3):, pp. 
179 - 189, 2000.

[23] Y-C Hu and D Johnson, "Ensuring Cache Freshness in On-Demand Ad Hoc Protocols.," In Proceedings of Int'l Workshop on Principles of Mobile Computing (POMC), pp. 25 - 30, 2002.

[24] D Johnson and D Maltz, "Dynamic Source Routing in Ad Hoc Wireless Networks.," In T. Imielinski and H. Korth, editors, MObile Comouting, Kluwer Academic, p. Chapter 5, 1996.

[25] $\mathrm{R}$ Ogier, $\mathrm{M}$ Lewis, and $\mathrm{F}$ Templin, "Topology Dissemination Based on Reverse-Path Forwarding (TBRPF).," Internet Engineering Task Force., 2003.

[26] Mohammed Ilyas, The Handbook of Ad Hoc Wireless Networks , Mohammed Ilyas, Ed. Boca Raton, Florida : CRC Press LLC, 2003.

[27] V Park and S Corson. (2001) IETF MANET Working Group. [Online]. http://tools.ietf.org/html/draft-ietfmanet-tora-spec-04

[28] A Nascimento, S Queiroz, E Mota, L Galvao, and E Nascimento, "Influence of Routing Protocol on VoIP Quality Performance in Wireless Mesh Backbone," in Second International Conference on Next Generation Mobile Applications Srvices and Technology, 2008, pp. 450-455.

[29] E Thibodeau, M Youssef, and A C Houle, "Investigating MANET Performance in a VoIP Context," International Journal of Engineering and Technology, pp. 920-923, 2006.

[30] Xiong Hong, Guo Yuan, and Zhu Fang, "Simple Voice over IP (VoIP) Implementation," EEL6586, pp. 1-12, 2003.

[31] R K Nadesh, D Sumathy, and M B Benjula Anbu Malar, "Performance Analysis of MANET(WLAN) using different Routing Protocols in multi service environments- An quantitative study," International Journal of Advanced Networking Applications, vol. 03, no. 02, pp. 1076-1079, 2011.

[32] A K Gupta, $H$ Sadawarti, and A K Verma, "Performance analysis of AODV, DSR \& TORA Routing Protocols," International Journal of Engineering and Technology, vol. 2, pp. 226-231, 2010.

[33] P Stuedi and G Alonso, "Wireless Ad Hoc VoIP," in Workshop on middle ware for Next-generation coverage Networks and Applications, 2007.

[34] V S Tiwari, S Mandal, C Sarkar, and K Das, "Study of VoIP Application in MANET on DSR Routing Protocol," Department of Computer Science and Engineering, Indian Institute of Technology, India, Thesis 2009.
[35] S Armania, L Gallucci, and S Palazzo, "Transmission of VoIP Traffic in multihop Ad hoc IEEE 802.11b Networks: Experimental Results," in First International Conference on Wireless Internet, 2005, pp. 148-155.

[36] L Atzori, F Boi, and G Nonnis, "IP Telephony over Mobile Ad Hoc Networks: Joint Routing and Playout Buffering," in IEEE International Conference on Communication, 2008, pp. 1861-1865.

[37] H M Chong and H S Mathews, "Comparative Analysis of Traditionsl Telephone and Voice-over-InternetProtocol (VoIP)," in International Conference record on IEEE and the Environment, 2004, pp. 106-111.

[38] H Y Zhang, M E Bialkowski, G A Einicke, and J Homer, "An Extended AODV Protocol for VoIP Application in Mobile Ad Hoc Network," Transactions on Electrical Engineering, Electronics and Communications ECTI, vol. 7, no. 2, 2009.

[39] X Jia, D Li, and D Du, "QoS Topology Control in Ad Goc Wireless Networks," in INFOCOM 2004. Twentythird AnnualJoint Conference of the IEEE Computer and Communications Societies, Hong Kong, 2004, pp. 1264-1272.

[40] R Rajaraman, "Topology control and routing in ad hoc networks: a survey," in Proceedings of the 16th International Distributed Processing Symposium, 2002, pp. 60-73.

[41] N S.M Usop, A Abdullah, and A F.A Abidin, "Performance Evaluation of AODV, DSDV \& DSR Routing Protocols in Grid Environment," International Journal of Computer and Network Security, vol. 9, no. 7, 2009.

[42] P K Suri and S Maan, "A Simulation Based QoS Constraint performance Evaluation of Routing Protocols in a MANET carrying packet Telephony," International Journal of Computer System, vol. 2, pp. 47-53, 2011.

[43] Anuj K Gupta, Harsh Sadawarti, and Anil K Verma, "Performance analysis of AODV, DSR \& TORA Routing Protocols," IACSIT International Journal of Engineering and Technology, vol. 2, no. 2, pp. 226-231, April 2010.

[44] B Vaidya, M K Denko, and J J.P.C Rodrigues, "Secure Framework for Voice Transmission Over Multipath Wireless Ad-Hoc Network," in Proceedings of the 28th IEEE conference on Global telecommunications (GLOBECOM), 2009, pp. 4299-4304.

[45] $U$ T Clausen and $R$ Cole, "MANET Network Management and Performance Monitoring for NHDP and OLSRV," in International Conference on Computer Network and Service Management (CNSM), 2010, pp. 290-293.

[46] S Garg and M Kappes, "Can I add a VoIP call?," in IEEE International Conference on Communications, 
2003, pp. 779-783.

[47] Lady Noreen P. Santos, "Voice Traffic over Mobile Ad Hoc Networks: A performance analysis of the Optimized Link State Routing Protocol (OLSR)," in ACI Academic Conference International , Cape Town, South Africa , 2009.

[48] L. N. P Santos, "Voice Traffic over MANET: A Performance analysis of the optimised link state routing protocol (OLSR)," Department of Air University, Air Force Institute of Technology, Ohir-India, Thesis 2009.

[49] Encyclopeadia, "Encyclopeadia on ad hoc and ubiquitous computing,". [Online]. http://books.google.co.uk/books?id=ueSFeCoNNP0C\& $\mathrm{pg}=$ PA588\&lpg $=$ PA588\&dq $=$ mobility + in + MANET + wi th+VoIP\&source $=$ bl\&ots $=$ u9gSTwESlm\&sig $=$ Ndk5gW bv5rT6MMujyMbEiml0thY\&hl=en\&sa=X\&ei=QH8pT SyMsGb8gOFpp3XAw\&ved=0CGwQ6AEwBw\#v=0 nepage\&q=mobility\%20in\%20MANET\%20with\%20V oIP

[50] Hoebeke Jeroen, Moeman Ingrid, Dhoedt Bart, and Demeester Piet, "An Overview of Mobile Ad Hoc Networks: Applications and Challenges," Ghent University, Ghent-Belgium, Research Paper.

[51] Minhas Qurratul_Ain, Mahmood Hasan, and Malik Hafiz, "The Role of Ad Hoc Networks in Mobile Telecommunication," Department of Electronics, Quadi-Azam University, Islamabad, Islamabad, Pakistan , Recent Developments in Mobile Communications - A Multidisciplinary Approach.

[52] Rajan Kumar. AuthorSTREAM. [Online]. http://www.authorstream.com/Presentation/rajaniter07165061-voip-ppt-rajan-seminar-jukta-sciencetechnology-powerpoint/

[53] E Fasolo, F Maguolo, A Zanella, and M Zorzi, "VoIP Communications in Wireless Ad Hoc Network with gateways," Journals on Computers and Communications, pp. 69-74, 2007.

[54] E Fasolo et al., "VoIP Communications in Wireless Ad hoc Network with Gateways," 12th IEEE Symposium on Data Grids, pp. 69-74, 2007. 